\title{
Curcumin attenuates hepatic fibrosis and insulin resistance induced by bile duct ligation in rats
}

\author{
Azam Eshaghian ${ }^{1}$, Ameneh Khodarahmi ${ }^{1}$, Fatemeh Safari ${ }^{2}$, Fariba Binesh ${ }^{3}$ and Ali Moradi ${ }^{1 *}$ \\ ${ }^{1}$ Department of Biochemistry, School of Medicine, Shahid Sadoughi University of Medical Sciences and Health Services, Yazd, \\ 8915173149, Iran \\ ${ }^{2}$ Department of Physiology, School of Medicine, Shahid Sadoughi University of Medical Sciences and Health Services, Yazd, \\ 8915173149, Iran \\ ${ }^{3}$ Department of Pathology, Shabid Sadoughi University of Medical Sciences, Yazd, 8915173149, Iran
}

(Submitted 5 November 2017 - Final revision received 14 March 2018 - Accepted 27 March 2018 - First published online 8 June 2018)

\section{Abstract}

Recent studies have strongly indicated the hepatoprotective effect of curcumin; however, the precise mechanisms are not well understood. This study aimed to determine the protective effect of curcumin on hepatic damage and hepatic insulin resistance in biliary duct ligated (BDL) fibrotic rat model. To accomplish this, male Wistar rats were divided into four groups (eight for each): sham group, BDL group, sham + Cur group and BDL + Cur group. The last two groups received curcumin at a dose of $100 \mathrm{mg} / \mathrm{kg}$ daily for 4 weeks. The mRNA/protein expression levels of Ras-related C3 botulinum toxin substrate 1 (Rac1), Rac1-GTP, dinucleotide phosphate oxidase 1 (NOX1), signal transducer and activator of transcription 3 (STAT3), suppressor of cytokine signalling 3 (SOCS3), insulin receptor substrate 1 (IRS1), extracellular signalregulated kinase 1 (ERK1), specific protein 1 (Sp1) and hypoxia-inducible factor-1 $\alpha$ (HIF-1 $\alpha$ ) were measured by real-time PCR and Western blotting, respectively. Fasting blood glucose, insulin and Leptin levels were determined and homoeostasis model assessment-estimated insulin resistance, as an index of insulin resistance, was calculated. Curcumin significantly attenuated liver injury and fibrosis, including amelioration of liver histological changes, reduction of hepatic enzymes, as well as decreased expression of liver fibrogenesis-associated variables, including Rac1, Rac1-GTP, NOX1, ERK1, HIF-1 $\alpha$ and Sp1. Curcumin also attenuated leptin level and insulin resistance, which had increased in BDL rats $(P<0.05)$. Furthermore, compared with the BDL group, we observed an increase in IRS1 and a decrease in SOCS3 and STAT3 expression in the curcumin-treated BDL group $(P<0.05)$, indicating return of these parameters towards normalcy. In conclusion, Curcumin showed hepatoprotective activity against BDL-induced liver injury and hepatic insulin resistance by influencing the expression of some genes/ proteins involved in these processes, and the results suggest that it can be used as a therapeutic option.

\section{Key words: Curcumin: Suppressor of cytokine signalling 3: Insulin receptor substrate 1: Liver fibrosis: Insulin resistance}

Bile duct ligation (BDL) is an experimental model used to induce obstructive cholestatic injury and ultimately liver fibrosis in rats ${ }^{(1)}$. Studies have shown that hepatic stellate cells (HSC) are directly involved in the process of fibrogenesis and that their activation is influenced by the formation of reactive oxygen species (ROS) and the presence of inflammatory mediators such as TNF- $\alpha$, IL, inducible nitric oxide synthase (iNOS) and $\mathrm{NF}-\kappa \mathrm{B}^{(2)}$. The presence of such factors leads to activation of quiescent HSC, which express large quantities of extracellular matrix (ECM) components. Mediators such as leptin, an adipose tissue adipokine, contribute to the activation of nicotinamide adenine dinucleotide phosphate oxidase (NOX) complex in activated $\mathrm{HSC}^{(3)}$. In general, leptin has profibrogenic action in
HSC that is applied through Janus kinase (JAK)-signal transducer and activator of transcription (STAT) pathway. Leptin induces JAK2 activation ${ }^{(4)}$. Activated JAK2 phosphorylates Rasrelated $\mathrm{C} 3$ botulinum toxin substrate 1 (Rac1) and consequently activates NOX complex ${ }^{(5)}$. Numerous stimuli such as cytokines and oxidative stress induce activation of Rac1 and formation of its biologically activated form, Rac1-GTP. Rac1-GTP induces the production of active NOX in a hepatic fibrosis model ${ }^{(6)}$. Among the NOX family, NOX1 seems to play a more crucial role in ROS generation in $\mathrm{HSC}^{(7)}$. Oxidative stress caused by the NOX1 activity finally induces the extracellular signal-regulated kinase 1 (ERK1) phosphorylation. These kinases phosphorylate transcription factors specific protein (Sp)-1 and 3, which in turn

Abbreviations: BDL, biliary duct ligated; DPPH, 2,2-diphenyl-1-picrylhydrazyl; ECM, extracellular matrix; ERK1, extracellular signal-regulated kinase 1; FBG, fasting blood glucose; HIF-1 $\alpha$, hypoxia-inducible factor- $1 \alpha$; HSC, hepatic stellate cells; HOMA-IR, homoeostasis model assessment-estimated insulin resistance; iNOS, inducible nitric oxide synthase; IR, insulin resistance; IRS1, insulin receptor substrate 1; JAK, Janus kinase; MDA, malondialdehyde; NOX1, dinucleotide phosphate oxidase 1; Rac1, Ras-related C3 botulinum toxin substrate 1; SOCS3, suppressor of cytokine signalling 3; Sp1, specific protein 1; ROS, reactive oxygen species; STAT3, signal transducer and activator of transcription 3 ; TGF- $\beta 1$, transforming growth factor- $\beta 1$. 
induces collagen I gene expression in $\mathrm{HSC}^{(4)}$. In recent years, studies showed that hypoxia-inducible factor- $1 \alpha$ (HIF- $1 \alpha$ ), which is induced by ERK1, was significantly increased in activated HSC. Therefore, the ERK/HIF- $1 \alpha$ signalling pathway is believed to play an important role in the genesis of progressive fibrosis ${ }^{(8)}$. An increase in transcriptional activity of HIF- $1 \alpha$ leads to upregulation of NF- $\kappa \mathrm{B}$, an inflammatory molecule, and transforming growth factor- $\beta 1$ (TGF- $\beta 1$ ), as the most potent stimulator of collagen biosynthesis in the liver. Activation of the HIF- $1 \alpha / \mathrm{NF}-$ $\kappa \mathrm{B}$ signalling pathway can increase TNF- $\alpha$, interleukins and iNOS expression ${ }^{(9)}$. In addition to HSC activation, these parameters, along with leptin, can inhibit insulin signalling in hepatocytes and induce hepatic insulin resistance (IR) ${ }^{(10)}$.

After leptin binds to its receptor, activated JAK2 phosphorylates STAT3, which in turn participates in leptin-induced expression of suppressors of cytokine signalling (SOCS) family of proteins ${ }^{(11)}$. SOCS proteins bind to their target molecules and degrade them through the ubiquitin pathway. Suppressor of cytokine signalling 3 (SOCS3) is a potent in vivo inhibitor of insulin receptor signalling ${ }^{(12,13)}$.

Insulin Receptor Substrate (IRS) family of proteins, as critical signalling molecules for insulin action, consist of four members: insulin receptor substrates 1 (IRS1) to IRS4. Hepatic IRS1 is closely linked to glucose homoeostasis ${ }^{(14)}$. The SOCS3 protein finally causes hepatic IR, a common pathological state, via several mechanisms such as promoting ubiquitin-mediated degradation of insulin receptor, IRS1 and other insulin signalling key molecules ${ }^{(15,16)}$.

According to the information mentioned above, IR parallels the process of liver fibrogenesis ${ }^{(17)}$. In hepatocytes, the IR ultimately leads to hyperinsulinaemia and fasting hyperglycaemia ${ }^{(18,19)}$.

Curcumin (diferuloylmethane), a biologically active polyphenolic component found in Curcuma longa plant, has for centuries been used as a medicinal plant in different countries ${ }^{(20)}$. There are many reports of its anti-inflammatory, anti-diabetic, liver-protective and anti-fibrosis activity ${ }^{(6)}$. Recent research has demonstrated that the antioxidant and anti-inflammatory properties of curcumin can explain its hepatoprotective effects ${ }^{(21)}$, but the precise mechanisms are not well characterised.

According to the evidence that hepatic IR as a common pathological state is associated with liver fibrogenesis and affects the expression of some genes/proteins, including STAT3, SOCS3 and IRS1, in this study we aimed to evaluate the effect of curcumin, as an antioxidant and anti-diabetic agent, on expression of these parameters and consequently on hepatic IR in BDL fibrotic rat model. We also measured serum levels of leptin, which stimulate fibrogenesis, and also calculated homoeostasis model assessment-estimated IR (HOMA-IR), as the index of IR, using measured fasting blood glucose (FBG) and insulin levels.

\section{Methods}

\section{Animals and experimental procedures}

Adult male Wistar rats (weighting 200-250 g; Pasteur Institute) were housed in an air-conditioned room at $25^{\circ} \mathrm{C}$ with $12 \mathrm{~h}$ light$12 \mathrm{~h}$ dark cycles and maintained on standard rat chow diet with free access to drinking water. All of the study's protocols followed the current ethical considerations of local ethics committee of animal use. A total of thirty-two rats were randomly divided into two groups: sham group and BDL group. Each group was separated into two subgroups for treatment with either curcumin at a dose of $100 \mathrm{mg} / \mathrm{kg}$ per d (Sigma Chemicals Co. Purity (HPLC) $>80 \%$ ) suspended in $5 \%$ carboxymethyl cellulose $(\mathrm{CMC})^{(22-26)}$ or the same volume/weight of the $5 \%$ CMC vehicle by oral gavage once a day from the day after surgery for $28 \mathrm{~d}$. BDL procedure was accomplished as described previously ${ }^{(27)}$. Concisely, under general anaesthesia by xylazine $(10 \mathrm{mg} / \mathrm{kg})$ and ketamine $(90 \mathrm{mg} / \mathrm{kg})$ intraperitoneally, the common bile duct was exposed by a midline abdominal incision under sterile conditions. It was then ligated in two places with silk thread and sectioned between the ligatures ${ }^{(28)}$ and Cefazolin antibiotic was used to prevent infection. At the end of the 4-week period, fasting blood samples were collected by puncturing the heart under deep anaesthesia and they were centrifuged at $3000 \boldsymbol{g}$ for $15 \mathrm{~min}$. The serum was isolated and kept at $-70^{\circ} \mathrm{C}$ for further experiments. Liver tissues were divided into three parts: one part was frozen in liquid $\mathrm{N}_{2}$ for RNA extraction, the second part was kept at $-70^{\circ} \mathrm{C}$ to make homogenised tissue for assessment of oxidative stress parameters and Western blot analyses and the last one fixed with $10 \%$ neutral formalin for histology.

\section{Histopathological evaluation}

The liver specimens were fixed in $10 \%$ neutral formalin individually, dehydrated in alcohol and embedded in paraffin and sections were stained with haematoxylin-eosin (H\&E). Lobular and portal inflammation, focal hepatocyte necrosis, ductular proliferation, portal and septal fibrosis were examined by a pathologist.

\section{Assessment of liver function}

The collected serum was examined for aspartate aminotransferase (AST), alanine aminotransferase (ALT), alkaline phosphatase (ALP), total bilirubin (TBIL) and direct bilirubin (DBIL) using standard animal diagnostic kits (Pars Azmon Diagnostic Co.) and an auto analyser (model BT3000; Biotechnica).

\section{Determination of malondialdehyde in liver tissues}

The levels of malondialdehyde (MDA), a biomarker of lipid peroxidation, were determined spectrophotometrically by measuring thiobarbituric acid-reactive substances ${ }^{(29)}$. A measure of $100 \mu \mathrm{g}$ of homogenised liver tissue supernatant was incubated with $15 \%$ TCA, $0.375 \%$ thiobarbituric acid, $0.25 \mathrm{M} \mathrm{HCl}$ and $6.8 \mathrm{~mm} 2$, 6-ditert-butyl-4-methylphenol for $60 \mathrm{~min}$ in a boiling water bath. After cooling, the mixtures were centrifuged at $3000 \mathrm{rpm}$ for $15 \mathrm{~min}$ and absorbance of the supernatant was recorded at $532 \mathrm{~nm}$. For standard curve, 1,1,3,3-tetraethoxypropane was used. The results were expressed as $\mathrm{nmol} / \mu \mathrm{g}$ protein of the tissue. Protein concentrations in the supernatants were determined by the Bradford assay. 


\section{Free-radical scavenging activity assay in liver tissues}

The free-radical scavenging activity of curcumin in liver tissues was measured by 2, 2-diphenyl-1-picryl-hydrazyl (DPPH) assay ${ }^{(30)}$. $\mathrm{DPPH}$, a stable free radical at room temperature, is reduced in the presence of an antioxidant molecule. Free-radical scavenging activity was measured by adding $20 \mu \mathrm{l}$ of homogenised liver tissue supernatant to $380 \mu \mathrm{l}$ of phosphate buffer $(\mathrm{pH} 7.4)$ and $400 \mu \mathrm{l}$ of DPPH in methanol. Protein concentrations in the supernatants were determined by the Bradford assay. Later, it was incubated for $30 \mathrm{~min}$ at room temperature. The absorbance of samples was taken $v$. Blank at $520 \mathrm{~nm}$, and percentage inhibition activity was calculated by $(\%)=\left(\left(\mathrm{A}_{0}-\mathrm{A}_{1}\right) / \mathrm{A}_{0}\right) \times 100 \%$, where $\mathrm{A}_{0}$ is the absorbance of the control reaction and $A_{1}$ is the absorbance in the presence of the sample ${ }^{(31)}$.

\section{Determination of homoeostasis model}

assessment-estimated insulin resistance index and leptin concentration

The level of serum glucose was determined by Trinder's method using a glucose assay kit (Pars Azmoon). Serum insulin concentration was determined using a sandwich enzyme immunoassay kit (Mercodia) according to the manufacturer's instructions. IR was calculated on the basis of fasting levels of plasma glucose and insulin, according to the HOMA-IR. HOMA-IR was determined by the following formula: (fasting plasma insulin $(\mathrm{mU} / \mathrm{l}) \times$ (fasting plasma glucose $(\mathrm{mmol} / \mathrm{l}) / 22.5)^{(32)}$. Serum leptin levels were measured using a sandwich enzyme immunoassay kit (ZellBio GmbH).

\section{Western blotting analysis}

Liver cell proteins were extracted in phosphate saline buffer (100 mm TRIS-HCl, pH 7·4, $150 \mathrm{~mm} \mathrm{NaCl}, 0 \cdot 1 \%$ SDS and 1\% NP-40; with protease-inhibitor cocktail, 1:100; Sigma) by homogenisation of tissue samples $(30 \mathrm{mg}$ ) followed by incubation on ice for $30 \mathrm{~min}$ and subsequent centrifugation at $15000 \mathrm{rpm}\left(4^{\circ} \mathrm{C}, 30 \mathrm{~min}\right)$. Protein concentrations in the supernatants were determined by the Bradford assay. Samples were mixed with $5 \times$ sample buffer (4:1) and heated in boiling water for $5 \mathrm{~min}$. Proteins ( $100 \mu \mathrm{g} /$ lane) were resolved on a $10 \%$ SDS polyacrylamide gel and transferred onto a nitrocellulose membrane. Ponceau-S staining was performed to assure correct blotting. The membranes were blocked with 5\% bovine serum albumin in TRIS-buffered saline ( $\mathrm{pH} 7.4$ ) with $0.05 \%$ Tween-20 (TBS/T) for $2 \mathrm{~h}$ at room temperature and probed with monoclonal mouse anti-Rac1-GTP (Newest Biosciences) and monoclonal rabbit anti-Rac1, polyclonal rabbit anti-NOX1, monoclonal rabbit anti-STAT3, polyclonal rabbit anti-IRS1, polyclonal rabbit anti-SOCS3 and polyclonal rabbit anti- $\beta$-actin primary antibody, as endogenous control, from Abcam, at $4^{\circ} \mathrm{C}$ overnight. After washing the blot in TBS/T three times, the membranes were incubated with goat anti-rabbit and anti-mouse IgG secondary antibody (1:2000) conjugated with horse radish peroxidase (Cell Signaling) for $2 \mathrm{~h}$ at room temperature. The predicted sizes for Rac1 (21 kDa), Rac1-GTP (22 kDa), NOX1 (71 kDa), STAT3 (85 kDa), IRS1 $(175 \mathrm{kDa}), \operatorname{SOCS} 3(30 \mathrm{kDa})$ and $\beta$-actin $(42 \mathrm{kDa})$ were checked using molecular-weight marker. After extensive washing in TBS/T, protein bands were visualised with an enhanced chemiluminescence reagent using hydrogen peroxide and luminol as substrates on a ChemiDoc system (GBOX, 680x; Syngene). Thereafter, specific bands were quantified densitometrically using ImageJ software (National Institutes of Health).

\section{Gene expression analysis by quantitative real-time PCR}

Total RNA was extracted from liver tissues using an EZ-10 Spin Column Total RNA Mini-Preps Kit from Bio Basic, according to the manufacturer's protocol. By measuring the absorbance, with an Epoch microplate spectrophotometer (BioTek Instrument), at $260 \mathrm{~nm}$, concentrations of RNA were identified and their purity was evaluated by $260 / 280 \mathrm{~nm}$ absorbance ratio (Eppendorf). Complementary DNA (cDNA) was synthesised from total RNA using the PrimeScript ${ }^{\mathrm{TM}}$ RT Reagent Kit (TakaRa). In brief, $1 \mu \mathrm{g}$ of total RNA was mixed with PrimeScript RT Enzyme Mix, $5 \times$ PrimeScript Buffer, Oligo dT Primer, Random hexamers and RNase-Free $\mathrm{dH} 2 \mathrm{O}$, as described in the manufacturer's protocol, in a total volume of $10 \mu \mathrm{l}$. The cDNA samples were diluted 1/10, and aliquots were frozen at $-20^{\circ} \mathrm{C}$ until the PCR was carried out. Real-time quantitative PCR (SYBR ${ }^{\circledR}$ Green PCR Master Mix kit; TakaRa) was performed in the Rotor Gene system (Corbett Research 2004). The programme was set to run for one cycle at $95^{\circ} \mathrm{C}$ for $10 \mathrm{~min}$, followed by forty cycles at $95^{\circ} \mathrm{C}$ for $30 \mathrm{~s}, 60^{\circ} \mathrm{C}$ for $30 \mathrm{~s}$ and $72^{\circ} \mathrm{C}$ for $30 \mathrm{~s}$. Normalisation was achieved against $\beta$-actin, as an internal control. Relative quantity of gene expression was analysed based on the $\Delta C_{t}$ method and the results were calculated as $2^{-\Delta \Delta C_{t}}$. The primers used in this study are listed in Table 1.

\section{Statistical analysis}

Data obtained from four groups were represented as the means and standard deviations. Normality was tested by the ShapiroWilk test. Differences between obtained values were carried out by one-way ANOVA followed by Tukey-Kramer multiple comparison using Graphpad Prism 6.01 software. The differences below 0.05 were considered statistically significant.

\section{Results}

Curcumin treatment attenuated bile duct ligation-induced histopathological changes

The histological examination of liver by Harris H\&E staining revealed that sham-operated control rats had normal liver morphology with intact hepatocytes and portal tracts (Fig. 1(a) and (b)). Parenchymal necrosis and an abundance of newly formed bile ducts, including bridging of the portal tracts, were found in BDL rats (Fig. 1(c)). Curcumin supplementation significantly amended all of the histopathological changes observed in the BDL group (Fig. 1(d)). It is inferred that curcumin treatment ameliorated BDL-induced liver fibrosis.

Effect of curcumin on indices of hepatic function in biliary duct ligated rats

Table 2 represents the effect of curcumin on markers of liver damage. The serum levels of AST, ALT, ALP and total and DBIL were significantly increased in BDL rats $(P<0 \cdot 05)$. The high 
Table 1. Primer sequences used for real-time PCR

\begin{tabular}{lllr}
\hline Genes & Forward primer (5'-3') & Reverse primer (5'-3') & Product size (bp) \\
\hline SOCS3 & GGGACCAAGAACCTACGC & GCTGCTCCTGAACCTCAAA & 188 \\
IRS1 & TGGATGCAAGTGGATGACTC & CGGAGGATTGTTGAGATGGT & 170 \\
STAT3 & GACGGAGAAGCAGCAGATG & ACGATCCTCTCCTCCAGC & 158 \\
ERK1 & TCCCTCTCAAGCTGCCACAT & ACATCCAATCACCCACACACA & 58 \\
HIF- $1 a$ & CTATGACGTGCTTGGTGCTGA & CTGTACTGTCCTGTGGTGACTT & 270 \\
Sp1 & CTGCAAGGGTCTGATTCTCTA & AGCTTGTCCACCTTGAACTA & 149 \\
collagen I & ATCAGCCCAAACCCCAAGGAG & CGCAGGAAGGTCAGCTGGATAG & 127 \\
$a-S M A$ & GCTCCATCCTGGCTTCTCTATC & GGGCCAGCTTCGTCATACTC & 72 \\
TGF- $\beta 1$ & AAGAAGTCACCCGCGTGCTA & TGTGTGATGTCTTTGTTTGTC & 69 \\
TNF- $a$ & ATGTGGAACTGGCAGAGGAG & CCACGAGCAGGAATGAGAAGAG & 98 \\
iNOS & TGGTCCAACCTGCAGGTCTTC & CAGTAATGGCCGACCTGATGTTG & 120 \\
NF-KB & TGTGAAGAAGCGAGACCTGGAG & GGCACGGTTATCAAAAATCGGATG & 204 \\
IL- $1 \beta$ & TACAAGGAGAGACAAGCAACGACA & GATCCACACTCTCCAGCTGCA & 119 \\
$\beta-$ Actin & CGTTGACATCCGTAAAGACCTC & AGCCACCAATCCACACAGA & 172 \\
\hline
\end{tabular}

SOCS3, suppressor of cytokine signalling 3; IRS1, insulin receptor substrate 1; STAT3, signal transducer and activator of transcription 3; ERK1, extracellular signal-regulated kinase 1 ; HIF-1a, hypoxia-inducible factor-1a; Sp1, specific protein 1 ; $a$-SMA, $a$-smooth muscle actin; TGF- $\beta 1$, transforming growth factor- $\beta 1$; iNOS, inducible nitric oxide synthase.
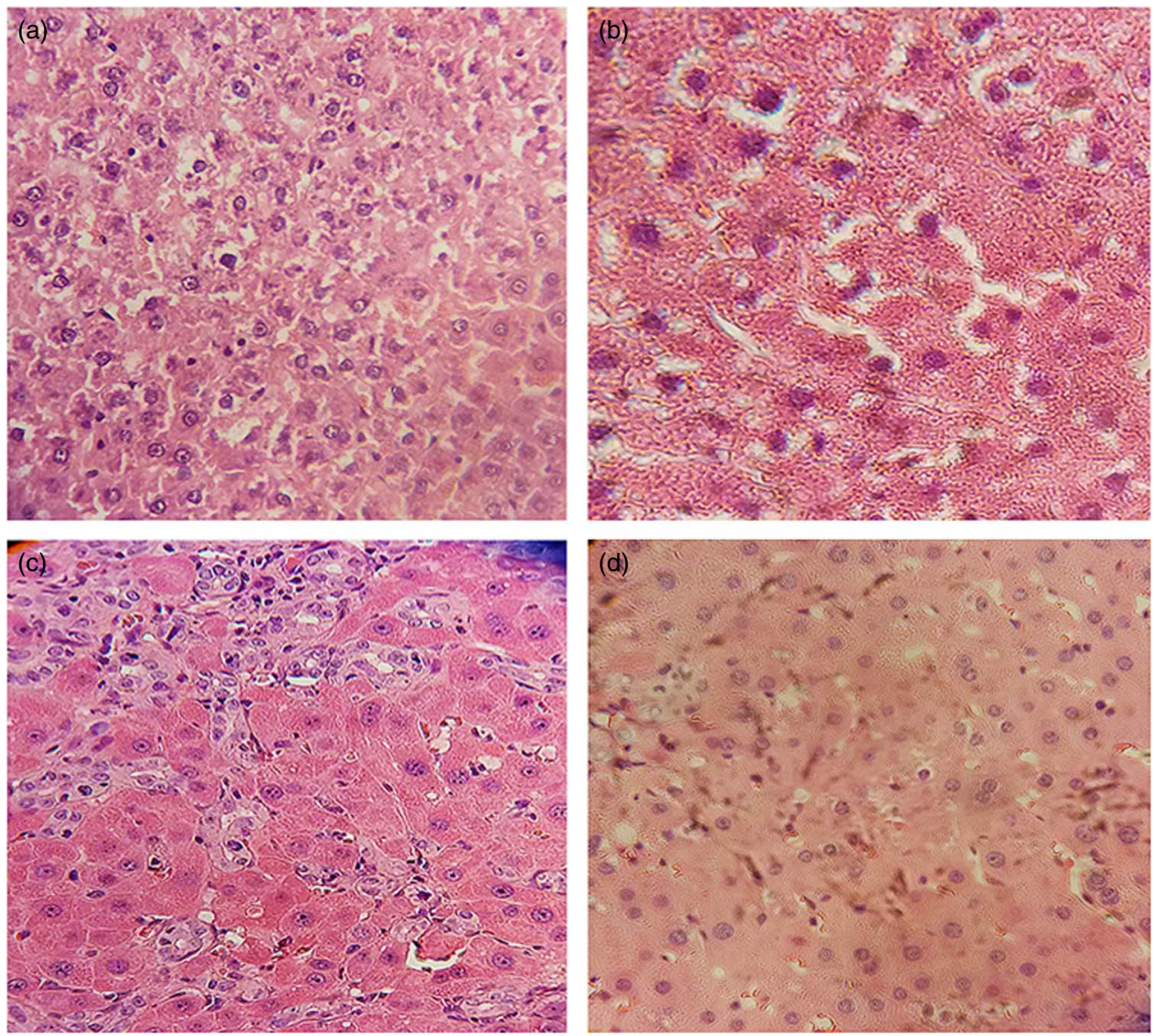

Fig. 1. Curcumin treatment reduced bile duct ligation (BDL)-induced liver injury, as shown by histological examination. Representative photomicrographs of haematoxylin-eosin staining showing (a) Sham, (b) Curcumin-treated Sham (Sham + Cur), (c) BDL and (d) Curcumin-treated BDL (BDL+Cur) rat liver sections.

level of these parameters was decreased in BDL rats after treatment with curcumin $(P<0 \cdot 05)$.

\section{Effect of curcumin on lipid peroxidation}

The effect of curcumin treatment on MDA levels in rat liver tissue of groups under investigation is shown in Table 2. The liver MDA level significantly increased in BDL rats compared with the sham group $(P<0 \cdot 05)$. It was found that curcumin could significantly decrease the formation of MDA in BDL-treated rats.

\section{Free-radical scavenging activity}

In this experiment, the total antioxidant capacity of liver tissue was measured by DPPH method (Table 2). DPPH-scavenging activity was decreased significantly in BDL rats compared with 
Table 2. Effect of curcumin on liver enzymes (aspartate aminotransferase (AST), alanine aminotransferase (ALT) and alkaline phosphatase (ALP)), total bilirubin (TBIL), direct bilirubin (DBIL), malondialdehyde (MDA) and 2,2-diphenyl-1-picrylhydrazyl (DPPH) in four groups of rats

(Mean values and standard deviations)

\begin{tabular}{|c|c|c|c|c|c|c|c|c|}
\hline & \multicolumn{2}{|c|}{ Sham } & \multicolumn{2}{|c|}{ Sham + Cur } & \multicolumn{2}{|c|}{ BDL } & \multicolumn{2}{|c|}{$\mathrm{BDL}+\mathrm{Cur}$} \\
\hline & Mean & SD & Mean & SD & Mean & SD & Mean & SD \\
\hline AST (U/I) & 1550 & $121 \cdot 6$ & 1380 & 147.0 & $4680^{*}$ & $258 \cdot 1$ & $3120 \dagger$ & 237.7 \\
\hline ALT (U/l) & 500 & 59.2 & 470 & 44.3 & $1470^{*}$ & $118 \cdot 2$ & $1260 \dagger$ & $32 \cdot 0$ \\
\hline $\operatorname{ALP}(\mathrm{U} / \mathrm{l})$ & 2540 & $162 \cdot 2$ & 2610 & $42 \cdot 0$ & $9470^{*}$ & $321 \cdot 7$ & $5430 \dagger$ & $541 \cdot 1$ \\
\hline TBIL (mg/l) & $2 \cdot 0$ & 0.0 & 1.9 & 0.0 & $110 \cdot 4^{*}$ & $16 \cdot 7$ & $73.5 \dagger$ & 7.8 \\
\hline DBIL (mg/l) & 0.3 & 0.0 & 0.4 & 0.0 & $64 \cdot 6^{\star}$ & 9.0 & $42.5 \dagger$ & 8.8 \\
\hline MDA (nmol/pg protein) & 7.32 & 0.33 & $7 \cdot 20$ & 0.51 & $22 \cdot 88^{\star}$ & 1.90 & $11 \cdot 70 \dagger$ & 0.74 \\
\hline DPPH (\% inhibition/mg tissue) & $6 \cdot 71$ & 0.46 & $7 \cdot 17$ & $1 \cdot 21$ & $2 \cdot 01^{*}$ & 0.18 & $4.19 \dagger$ & 0.83 \\
\hline
\end{tabular}

Cur, curcumin; BDL, bile duct ligation.

${ }^{*} P<0.05$, compared with the sham group.

$\dagger P<0.05$, compared with the BDL group.

Table 3. Comparison of $a$-smooth muscle actin ( $\alpha$-SMA), transforming growth factor $\beta 1$ (TGF- $\beta 1$ ), collagen I, IL-1 $\beta$, TNF- $\alpha$, inducible nitric oxide synthase (iNOS) and NF-kB mRNA expression in liver tissue of four groups (Mean values and standard deviations)

\begin{tabular}{|c|c|c|c|c|c|c|c|c|}
\hline & \multicolumn{2}{|c|}{ Sham } & \multicolumn{2}{|c|}{ Sham + Cur } & \multicolumn{2}{|c|}{ BDL } & \multicolumn{2}{|c|}{$\mathrm{BDL}+\mathrm{Cur}$} \\
\hline & Mean & SD & Mean & SD & Mean & SD & Mean & SD \\
\hline$a-S M A$ & 3.54 & 0.07 & 4.01 & 0.14 & $6 \cdot 60^{*}$ & 0.10 & $3.92 \dagger$ & 0.39 \\
\hline TGF- $\beta 1$ & $4 \cdot 19$ & 0.13 & $3 \cdot 81$ & 1.22 & $6 \cdot 30^{*}$ & 0.01 & $3.55 \dagger$ & 1.00 \\
\hline Collagen I & $2 \cdot 70$ & 0.28 & 2.54 & 0.37 & $4 \cdot 30^{\star}$ & 1.02 & $2.41 \dagger$ & 0.31 \\
\hline $\mathrm{IL}-1 \beta$ & 1.00 & $0 \cdot 10$ & 1.13 & 0.07 & $2 \cdot 36^{*}$ & 0.18 & $1.54 \dagger$ & 0.19 \\
\hline TNF- $a$ & 1.02 & 0.23 & 1.06 & 0.12 & $3.70^{*}$ & 0.53 & $2.42 \dagger$ & 0.50 \\
\hline iNOS & 3.29 & 0.35 & 2.86 & 0.29 & $5 \cdot 90^{*}$ & 0.68 & $4.37 \dagger$ & 0.58 \\
\hline NF- $k B$ & 2.39 & 0.61 & 2.44 & 0.37 & $6 \cdot 11^{*}$ & 1.07 & $4.22 \dagger$ & 0.68 \\
\hline
\end{tabular}

Cur, curcumin; BDL, bile duct ligation.

* $P<0.05$, compared with the sham group.

$\dagger P<0.05$, compared with the BDL group.

Table 4. Effects of curcumin (Cur) on fasting blood glucose (FBG), fasting blood insulin (FBI), homoeostasis model assessmentestimated insulin resistance (HOMA-IR) index and leptin levels in serum of four groups (Mean values and standard deviations)

\begin{tabular}{|c|c|c|c|c|c|c|c|c|}
\hline & \multicolumn{2}{|c|}{ Sham } & \multicolumn{2}{|c|}{ Sham + Cur } & \multicolumn{2}{|c|}{$\mathrm{BDL}$} & \multicolumn{2}{|c|}{$\mathrm{BDL}+\mathrm{Cur}$} \\
\hline & Mean & SD & Mean & SD & Mean & SD & Mean & SD \\
\hline $\mathrm{FBG}(\mathrm{mmol} / \mathrm{l})$ & $5 \cdot 12$ & 0.18 & 5.35 & 0.49 & $14.53^{*}$ & 1.69 & $8.63 \dagger$ & 1.22 \\
\hline $\mathrm{FBI}(\mathrm{mU} / \mathrm{l})$ & $10 \cdot 16$ & 0.18 & $10 \cdot 25$ & 0.65 & $12 \cdot 44^{*}$ & 0.48 & $11.23 \dagger$ & 0.39 \\
\hline HOMA-IR & $2 \cdot 31$ & 0.08 & 2.42 & 0.09 & $8.04^{*}$ & $1 \cdot 15$ & $4.32 \dagger$ & 0.75 \\
\hline Leptin (ng/ml) & 5.55 & 0.24 & $5 \cdot 33$ & 0.55 & $8 \cdot 08^{\star}$ & 1.24 & $7.42 \dagger$ & 0.94 \\
\hline
\end{tabular}

$\mathrm{BDL}$, bile duct ligation.

${ }^{*} P<0.05$ compared with the Sham group.

$\dagger P<0.05$ compared with the BDL group.

the sham group $(P<0.05)$, and curcumin significantly increased this parameter in BDL-treated rats compared with the BDL group $(P<0.05)$.

\section{Effect of curcumin on mRNA expression of fibrotic markers and inflammatory mediators}

We measured the mRNA expression level of $\alpha$-smooth muscle actin $(\alpha-S M A), T G F-\beta 1$ and collagen $I$ as fibrotic markers and $I L-1 \beta, T N F-\alpha, i N O S$ and $N F-\kappa B$ as inflammatory mediators in liver tissue of groups under investigation by the quantitative PCR (qPCR) method (Table 3). We observed that expression levels of these genes were significantly increased in liver tissue of BDL rats compared with the sham group $(P<0.05)$.
However, treatment with curcumin reduced them in BDLtreated rats.

\section{Effect of curcumin on homoeostasis model assessment-estimated insulin resistance index and leptin concentration}

The data presented in Table 4 revealed a significant increase in FBG, fasting blood insulin (FBI), HOMA-IR index and leptin concentration in BDL rats compared with the sham group $(P<0.05)$. However, FBG, insulin, HOMA-IR index and leptin levels in BDL rats treated with curcumin were significantly decreased $(P<0.05)$ compared with the BDL group. 
Effects of curcumin on protein expression of Ras-related C3 botulinum toxin substrate 1, Ras-related C3 botulinum toxin substrate 1-GTP, dinucleotide phosphate oxidase 1, signal transducer and activator of transcription 3, suppressor of cytokine signalling 3 and insulin receptor substrate 1

To examine the anti-fibrotic effects of curcumin, protein expression of Rac1, Rac1-GTP and NOX1 (as fibrogenic proteins) and STAT3, SOCS3 and IRS1 (as proteins involved in the process of hepatic IR) in liver tissue of groups under investigation were assayed using Western blotting method. Results were normalised to $\beta$-actin expression. We observed significant increases in hepatic protein expressions of Rac1, Rac1-GTP, NOX1, STAT3 and SOCS3 and decrease in protein expressions of IRS1 in liver tissue of BDL rats compared with the sham group $(P<0.05)$. The expression levels of the first five proteins are decreased and the expression of IRS1 increased in liver tissue of BDL-treated rats compared with the BDL group $(P<0.05)$, as shown in Fig. 2 .

\section{Effects of curcumin on mRNA expression of signal} transducer and activator of transcription 3, suppressor of cytokine signalling 3, insulin receptor substrate 1, extracellular signal-regulated kinase 1, hypoxia-inducible factor- $1 \alpha$ and specific protein 1

After $28 \mathrm{~d}$ from BDL surgery and treatment with curcumin, we measured the mRNA expression levels of ERK1, HIF-1 $\alpha$ and Sp1 (as fibrogenic genes), as well as STAT3, SOCS3 and IRS1 (as genes involved in the process of hepatic IR), in liver tissue of groups under investigation by the qPCR method. Results were normalised with respect to $\beta$-actin as a housekeeping gene and were reported as expression relative units. We observed that ERK1, HIF-1 $\alpha$, Sp1, STAT3 and SOCS3 gene expressions significantly increased and IRS1 gene expressions decreased in liver tissue of $\mathrm{BDL}$ rats compared with the sham group $(P<0.05)$. However, treatment with curcumin reduced gene expression of ERK1, HIF-1 $\alpha, S p 1$, STAT3 and SOCS3 and enhanced gene expression of IRS 1 in the liver tissue of the BDLtreated group compared with the BDL group $(P<0.05)$, as shown in Fig. 3.

\section{Discussion}

Chronic liver damage can lead to hepatic fibrosis, a complex process caused by accumulation of ECM in the liver, as well as hepatic IR, a pathophysiological feature of hepatogenous diabetes. Hepatic fibrosis is the final pathway that may result in cirrhosis, which can ultimately require liver transplanta$\operatorname{tion}^{(17,33)}$, and hepatogenous diabetes is a common complication of liver cirrhosis and directly relates to loss of liver function $^{(34)}$.

BDL is a well-known model of cholestatic liver disease resulting in bile acid overload, an inflammatory stimulus, which causes oxidative damage and ultimately liver fibrosis and cirrhosis in rats $^{(1)}$. After BDL, ROS and inflammatory (a)
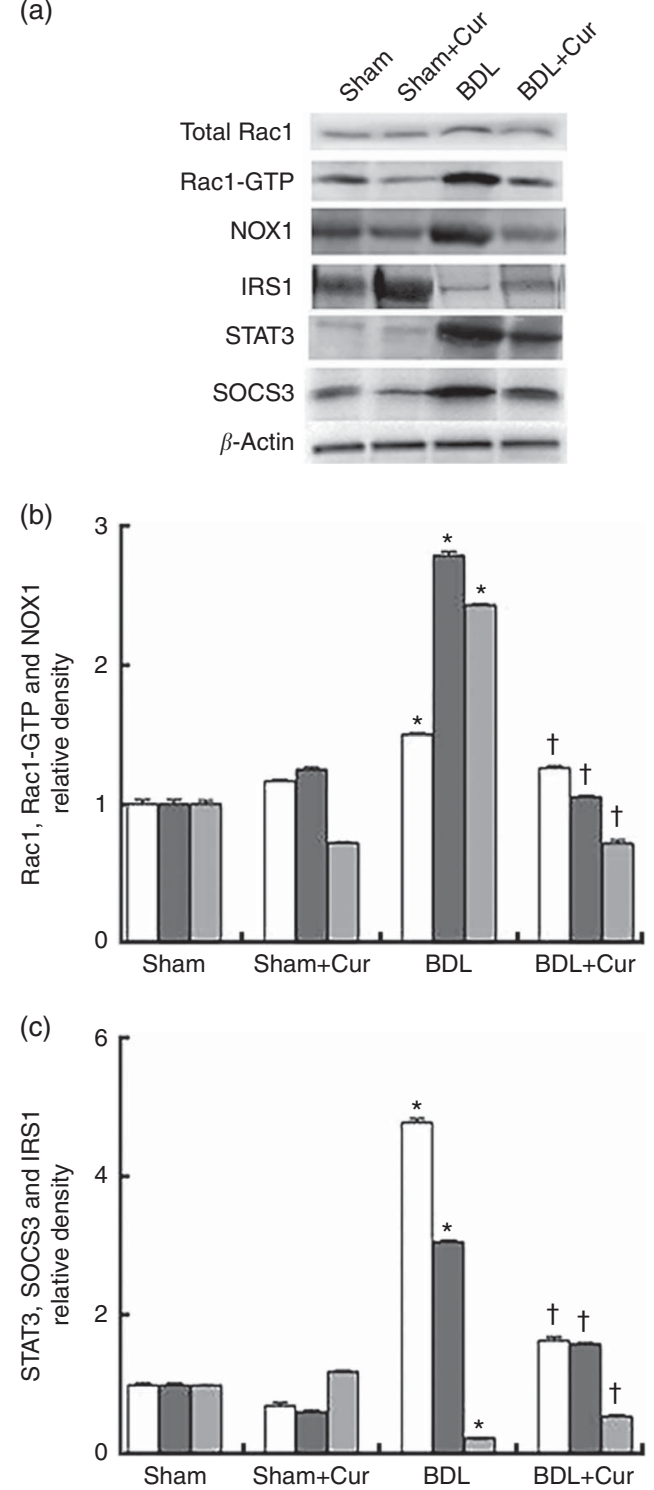

Fig. 2. Western blotting pattern of Ras-related C3 botulinum toxin substrate 1 (Rac1), Rac1-GTP, dinucleotide phosphate oxidase 1 (NOX1), signal transducer and activator of transcription 3 (STAT3), suppressor of cytokine signalling 3 (SOCS3) and insulin receptor substrate 1 (IRS1) protein expression (a); the relative density of protein expression levels of NOX1, Rac1 and Rac1-GTP, which are involved in ROS generation and liver fibrogenesis, (b) and STAT3, SOCS3 and IRS1, which contribute to insulin resistance (c) in the four studied groups (Sham, Curcumin-treated Sham (Sham + Cur), bile duct ligation (BDL), Curcumin-treated $\mathrm{BDL}(\mathrm{BDL}+\mathrm{Cur})$ ). Values are means $(n 6)$ and standard deviations represented by vertical bars. ${ }^{*} P$ value $<0.05$ compared with the Sham group. $\dagger P$ value $<0.05$ compared with the BDL group. b: $\square$, Rac1; $\square$, Rac1-GTP; $\square$, NOX1; c: $\square$, STAT3; $\square$, SOCS3; $\square$, IRS1.

mediators such as TNF- $\alpha$, interleukins, iNOS, NF- $\kappa \mathrm{B}$ and leptin, which are involved in liver fibrogenesis, in addition to HSC activation, inhibit insulin signalling in hepatocytes and induce hepatic $\mathrm{IR}^{(10)}$

This study aimed to investigate the effect of curcumin on the process of hepatic IR by evaluation of expression levels of STAT3, SOCS3 and IRS1 and also on hepatic fibrogenesis induced by BDL through assessment of expression profile of Rac1, Rac1-GTP, NOX1, Sp1, ERK1 and HIF-1 $\alpha$ in BDL rat 
(a)

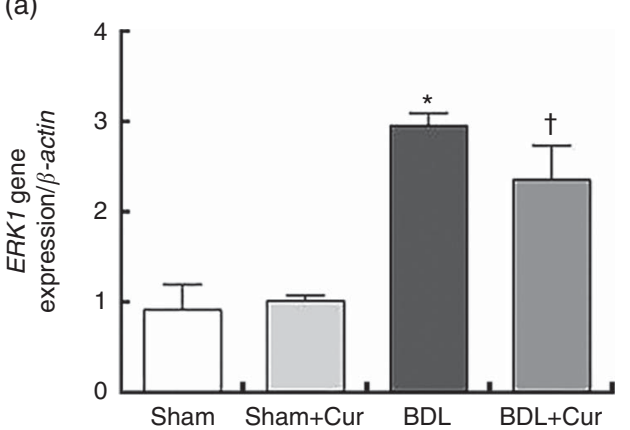

(c)

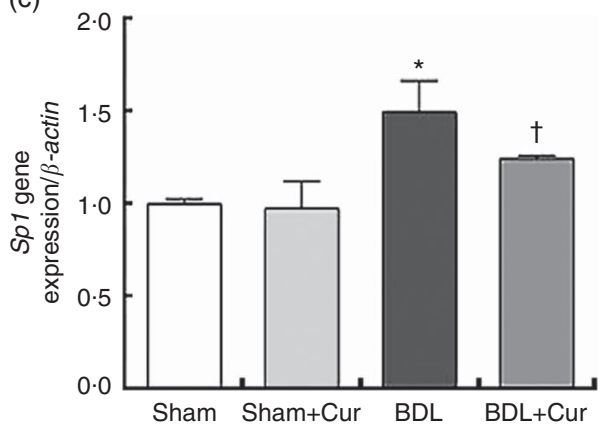

(e)

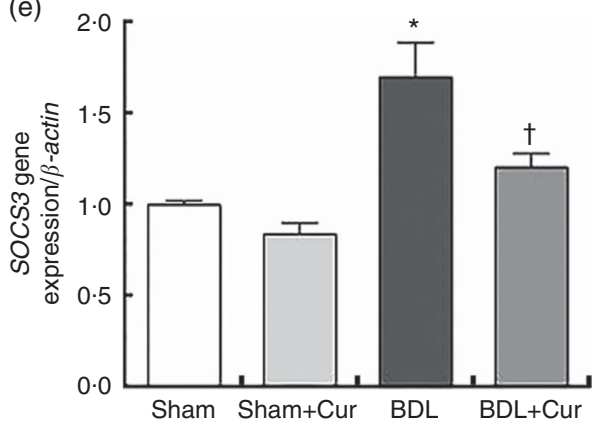

(b)

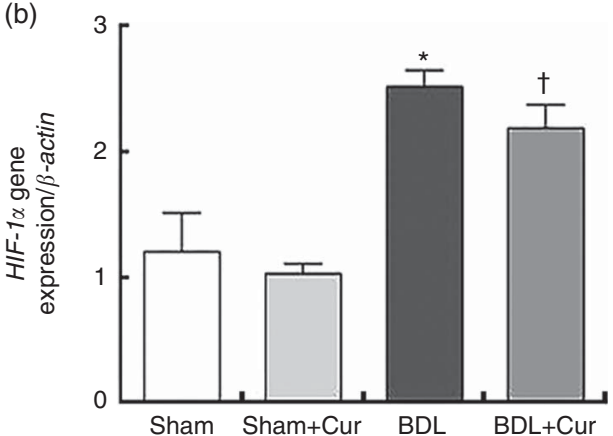

(d)

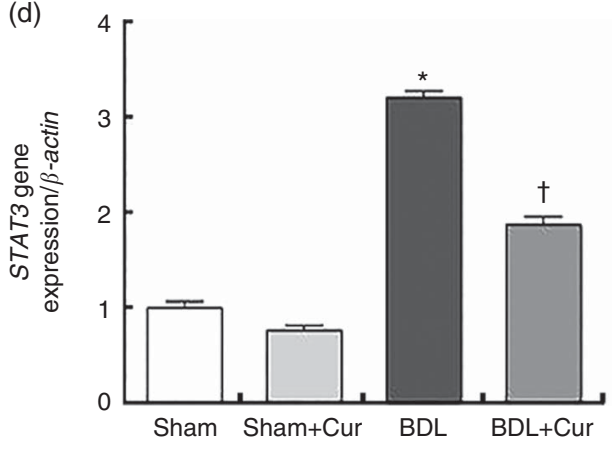

(f)

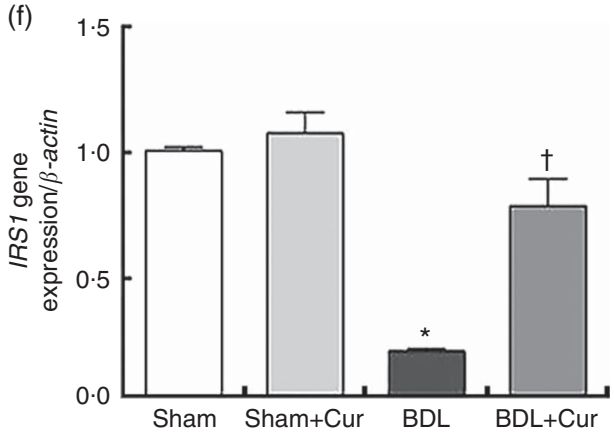

Fig. 3. Gene expression of extracellular signal-regulated kinase 1 (ERK1) (a), hypoxia-inducible factor-1a (HIF-1a) (b), specific protein 1 (Sp1) (c), signal transducer and activator of transcription 3 (STAT3) (d), suppressor of cytokine signalling 3 (SOCS3) (e) and insulin receptor substrate 1 (IRS1) (f) in the four studied groups (Sham, Curcumin-treated Sham (Sham + Cur), bile duct ligation (BDL), Curcumin-treated BDL (BDL +Cur)). * $P$ value $<0.05$ compared with the Sham group. † $P$ value $<0.05$ compared with the BDL group.

models. The hepatoprotective effect of curcumin was investigated through the evaluation of oxidative stress indicators, hepatic enzymes, hepatic fibrosis markers and leptin levels. To confirm IR, FBG and insulin levels were measured and HOMA-IR, as index of IR, was calculated.

Curcumin (diferuloylmethane) is a biologically active polyphenol derived from turmeric plant. It has beneficial properties such as anti-inflammatory, anti-diabetic, liver-protective and antifibrosis activity ${ }^{(20)}$. The effects of curcumin on the prevention of hepatic fibrogenesis have been confirmed ${ }^{(21)}$. Nevertheless, its molecular mechanism remains to be elucidated.

To the best of our knowledge, this is the first study that evaluates the efficacy of curcumin on hepatic IR through assessment of expression levels of STAT3, SOCS3 and IRS1 in BDL rats. Our findings manifested that curcumin treatment attenuated hepatic IR through down-regulation of either mRNA or protein expression of SOCS3 and STAT3, as well as upregulation of IRS1. In addition, curcumin reduced oxidative stress and subsequently ROS generation by down-regulation of Rac1, Rac1-GTP and NOX1, which in turn leads to attenuation of liver fibrosis through reduction of ERK1, HIF-1 $\alpha$ and Sp1 expression levels. To evaluate the fibrogenesis process during BDL, we measured mRNA expression of three fibrotic markers, including $\alpha-S M A, T G F-\beta 1$ and collagen $I$, as confirmatory tests, in liver tissue of four study groups. Compared with the sham group, BDL rats showed a significant increase in the fibrosis markers, but treatment of these rats with curcumin led to reduction in these markers.

During liver fibrogenesis, activated HSC secrete large quantities of collagen fibrosis and induce the accumulation of ECM leading to liver fibrogenesis ${ }^{(1)}$. We used the BDL method to induce liver fibrosis, and then we confirmed this process by evaluation of $\alpha$-SMA, TGF- $\beta 1$ and collagen I mRNA expression levels, as well as H\&E staining of hepatic tissue sections in BDL rats. To assess liver function, hepatic enzymes (AST, ALT and ALP), TBIL and DBIL were measured. On the basis of our 
findings, curcumin treatment of BDL rats significantly improved liver fibrosis and its function.

The oxidative stress can leads to lipid peroxidation, which accelerates collagen synthesis by stimulating HSC, as well as free-radical production. HSC are activated by MDA, a lipid peroxidation marker, and this activation is blocked by antioxidants $^{(35)}$. To assess the oxidative damage caused by BDL and the ability of curcumin to scavenging free radicals, we measured the MDA level and DPPH radical scavenging activity in hepatic tissue of four groups, respectively. Our findings have shown that the MDA level was increased significantly when exposed to oxidative stress caused by BDL and DPPH reduction was reduced in this status. In agreement with our findings, recent observations have shown an increase in MDA levels and decrease in DPPH reduction in fibrotic rats ${ }^{(36)}$. Svegliati Baroni et al. ${ }^{(37)}$ showed that fibrosis caused by lipid peroxidation and its products decreases after the administration of antioxidants in animal models. Our experiment demonstrated an increase in the DPPH radical scavenging activity and decrease in the MDA level, both of which confirm the protective effect of curcumin against oxidative stress caused by fibrosis in BDL rats.

Liver is a dynamic organ with fundamental contributions in detoxification, biosynthesis, metabolism and elimination of endogenous and exogenous substances. Its metabolic activity such as regulation of plasma glucose is tightly controlled by insulin and other metabolic hormones ${ }^{(38)}$.

Hepatic fibrogenesis is a pathologic process characterised by synthesis, secretion and accumulation of ECM derived from activated HSC in the liver ${ }^{(39)}$. As mentioned above, BDL process causes oxidative damage in hepatic tissue. Damaged hepatocytes produce inflammatory cytokines such as $I L-1 \beta, T N F-\alpha$, $i N O S$ and $N F-\kappa B$, which in turn increase the mRNA expression level of leptin that is an adipose tissue cytokine ${ }^{(40)}$. In our experiment, the mRNA expression levels of $I L-1 \beta, T N F-\alpha$, iNOS and $N F-\kappa B$ were remarkably increased in BDL fibrotic rats, and this effect was notably abolished by curcumin. In agreement with our findings, Reyes-Gordillo et al. ${ }^{(41)}$ determined that CCl4-administered rats depicted significant increases in TNF- $\alpha$, IL- 6 and IL- $1 \beta$ production and NF- $\kappa \mathrm{B}$ activation, whereas curcumin significantly suppressed these mediators of inflammation in liver damage. Their findings suggest that curcumin prevents liver damage by at least two mechanisms: acting as an antioxidant and by inhibiting NF- $\kappa \mathrm{B}$ activation and thus production of proinflammatory cytokines.

Leptin has profibrogenic and proinflammatory actions. Its profibrogenic action in HSC is applied through the JAK-STAT pathway. Leptin binding to its receptor induces Jak2 activation. Activated JAK2 subsequently phosphorylates Rac1 ${ }^{(5,33)}$. Rac1, a key mediator for accumulation of ECM by activated HSC, is associated with fibrogenesis. Its activated form (Rac1-GTP) stimulates NOX, multi-protein complexes that produce ROS in response to numerous stimuli. Among the NOX family, NOX1 seems to play a more crucial role in ROS generation in $\mathrm{HSC}^{(7)}$. NOX1 activity finally induces the phosphorylation of ERK1, an important marker in fibrogenesis that elevates the expression of transcription factor HIF- $1 \alpha$ and increases its activity by phosphorylation. HIF- $1 \alpha$ is significantly increased in hepatic fibrosis and activated HSC and subsequently targets gene families that represent profibrotic mediators, and thus plays an important role in the genesis and progression of tissue fibrosis ${ }^{(8)}$. ERK1 also phosphorylates Sp1, a critical transcription factor for collagen I expression in HSC. ERK1-dependent activation of Sp1 might be a consequence of the oxidative stress provoked by leptin. Generally, leptin is a stimulatory factor for gene expression and protein production of collagen $\mathrm{I}$; therefore, leptin may play an important role in the development of liver fibrosis ${ }^{(5)}$. Recent observations have demonstrated that in a BDL rat model circulating levels of leptin were increased ${ }^{(42)}$. Vivoli et $a l .{ }^{(33)}$ indicated that the absence of leptin or impaired leptin signalling resulted in a reduction of fibrogenesis in animals exposed to carbon tetrachloride. On the other hand, some studies have shown that in CCl4-fibrotic rats, the Rac1 activity led to increased ROS generation by NOX1, as well as increased activity of HSC, which ultimately intensified hepatic fibrosis ${ }^{(43)}$. In addition, Rac1 deficiency resulted in suppression of NOX1 and also reduction in oxidative stress ${ }^{(44)}$. Our observations showed that NOX1 expression level in BDL rats significantly increased, which was accompanied by an increase in Rac1 expression and, consequently, in Rac1-GTP, as well as an increase in leptin level, compared with the sham group. However, these rates were decreased after treatment of BDL rats with curcumin (BDL group compared with BDL + Cur). On the other hand, our findings have shown that expression of $H I F-1 \alpha$, ERK1 and Sp1, which play important roles in the genesis of progressive fibrosis, significantly increased in BDL rats compared with the sham group and treatment of the BDL group with curcumin reduced these variables. In agreement with our findings, Zhao et $a l .{ }^{(8)}$ have reported an increase in the expression of HIF- $1 \alpha$ and ERK in CCl4-fibrotic rats, but a decrease in their expression in the curcumin-treated group.

As already mentioned, leptin binding to its receptor induces activation of JAK2. In addition to Rac1, JAK2 can phosphorylate STAT3, which in turn dimerises and translocates to the nucleus and leads to increased transcription and expression of some genes such as SOCS family of proteins ${ }^{(12)}$. These proteins bind to phosphotyrosine residues present on their target molecules and induce their degradation through the ubiquitin pathway. Actually, SOCS proteins can act as ubiquitin ligases, facilitating the polyubiquitination and degradation of signalling proteins. Among the members of this family, SOCS3 is a potent in vivo inhibitor of insulin receptor signalling ${ }^{(13)}$

Insulin is the essential hormone involved in glucose homoeostasis, which exerts many biological effects through receptormediated tyrosine phosphorylation of the IRS family ${ }^{(19)}$. The members of this protein family, IRS1 to IRS4, are critical signalling molecules for insulin action. Among them, hepatic IRS1 closely linked to glucose homoeostasis ${ }^{(14)}$. The SOCS proteins, which up-regulated through the leptin/JAK2/STAT3 pathway, interfere with insulin signalling through ubiquitin-mediated degradation of IRS1/2 and other insulin signalling key molecules via the ubiquitin-proteasome system. In fact, overexpression of SOCS1 and 3 in the mouse liver causes $\mathrm{IR}^{(16)}$. One study has shown that $\mathrm{CCl} 4$ administration results in hepatotoxicity by alteration of IL-6/STAT3 pathway gene expression, and rutin, as a flavonoid found in a wide variety of plants, 


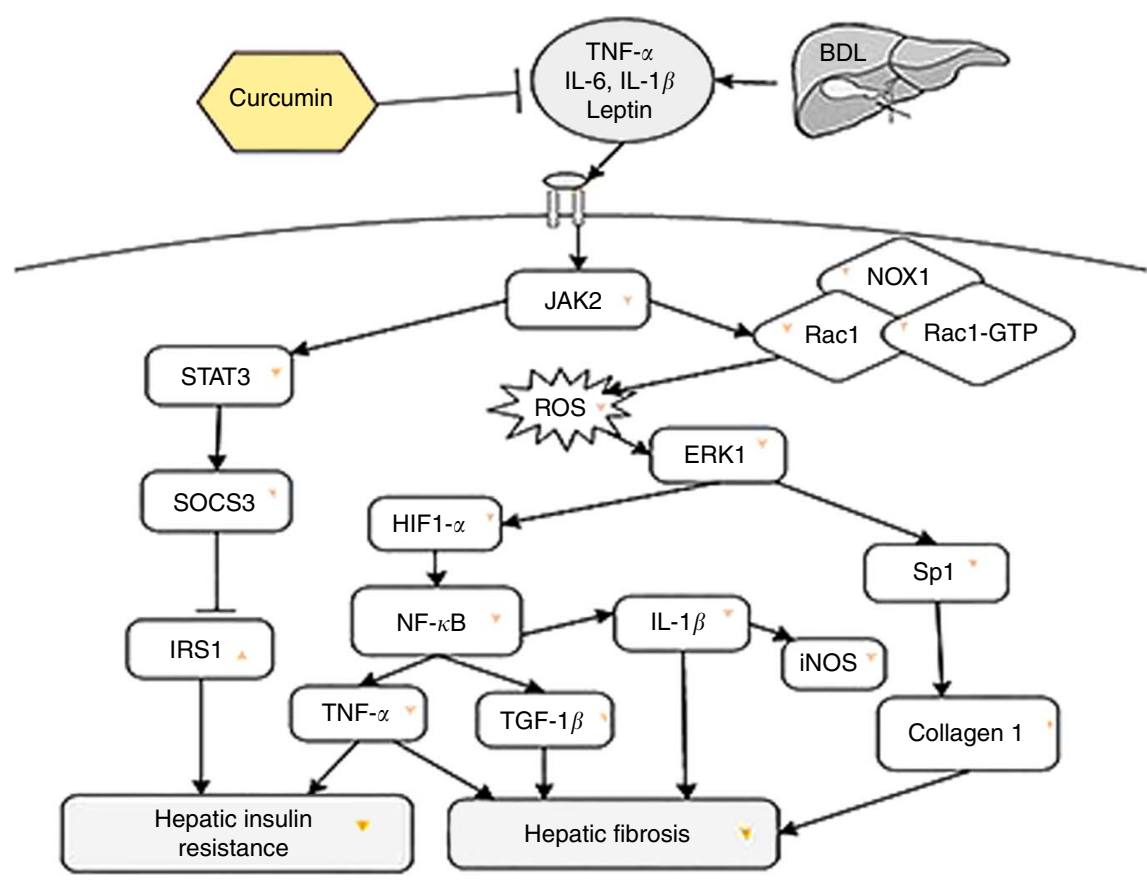

Fig. 4. The beneficial effect of curcumin against bile duct ligation (BDL)-induced liver fibrosis and hepatic insulin resistance is presented. This schematic diagram shows the effect of curcumin on intracellular molecules involved in these two pathological states in rats. JAK2, Janus kinase 2; STAT3, signal transducer and activator of transcription 3; NOX1, dinucleotide phosphate oxidase 1; RAc1, Ras-related C3 botulinum toxin substrate 1; ROS, reactive oxygen species; ERK1, extracellular signal-regulated kinase 1; SOCS3, suppressor of cytokine signalling 3; HIF-1, hypoxia-inducible factor-1; Sp1, specific protein 1; IRS1, insulin receptor substrate 1; TGF-1 $\beta$, transforming growth factor- $1 \beta ; \rightarrow$, induction or activation, - , inhibition or blockade; $\vee$ or $A$ indicates the effect of curcumin on protein/gene expression.

protects against $\mathrm{CCl} 4$-induced hepatotoxicity by reversing these expression changes ${ }^{(45)}$.

The result of another study showed that IL-6 is a major stimulus leading to IR, and metformin directly represses the IL-6-induced STAT3-SOCS3 pathway and improves hepatic $\mathrm{IR}^{(46)}$. Rui et al. ${ }^{(15)}$ showed that SOCS3 promotes ubiquitination and degradation of IRS1 in both cultured cells and animal tissues, contributing to IR. Hence, SOCS-mediated degradation of IRS protein via the ubiquitin-ligase pathway might be a general mechanism for inflammation-induced IR. Our observations showed that mRNA and protein expression level of STAT3 and SOCS3 in BDL rats significantly increased, and this increase was parallel with a decrease in the expression of IRS1 compared with the sham group. However, the expression level of STAT3 and SOCS3 was decreased and expression of IRS1 was increased upon treatment of BDL fibrotic rats with curcumin (BDL group compared with BDL + Cur).

According to the information mentioned, hepatic IR is associated with the development and progression of liver fibrosis ${ }^{(17)}$. In hepatocytes, the IR refers to increased demand for insulin to maintain glucose homoeostasis, which leads to persistent stimulation of insulin production by pancreatic B-cells and subsequently, hyperinsulinaemia ${ }^{(18)}$, as well as failure of insulin to inhibit hepatic glucose production, which is responsible for development of hyperglycaemia ${ }^{(47)}$. Sadek et al. ${ }^{(48)}$ revealed that CCl4 significantly increases oxidative stress, expression of the profibrotic cytokine genes such as TNF- $\alpha$ and TGF- $\beta$, blood glucose and insulin levels and IR index (HOMA-IR). However, these effects were abolished by camel milk either alone or in combination with bee honey because of their antioxidant properties.
Our findings have shown that FBG, FBI and HOMA-IR, as the index of IR, increased in BDL rats compared with the sham group and curcumin treatment of BDL group reduced these parameters (BDL group compared with BDL + Cur).

In short, BDL can lead to hepatic fibrosis on the one hand and hepatic IR on the other, by creating oxidative stress conditions and increasing inflammatory mediators in rats. According to our observation, a schematic diagram has been presented, which briefly explains the inhibitory effect of curcumin on liver fibrosis and hepatic IR in BDL rats (Fig. 4). Our findings demonstrated that curcumin treatment of BDL rats attenuated hepatic IR through down-regulation of SOCS3 and STAT3 and also up-regulation of IRS1. Furthermore, curcumin reduced inflammatory cytokines (IL- $1 \beta$, TNF- $\alpha$, iNOS, NF- $\kappa$ B and leptin), as well as oxidative stress, and subsequently ROS generation via down-regulation of Rac1, Rac1-GTP and NOX1, which in turn leads to attenuation of liver fibrosis through reduction of ERK1, HIF-1 $\alpha$ and Sp1 expression levels.

\section{Acknowledgements}

The results described in this paper were extracted from a Master's thesis, and the authors thank Misses Hafizi and Ajdari for research support.

This article was supported by Shahid Sadoughi University of Medical Sciences, Yazd, Iran.

All authors contributed to the literature search, analysis of the data published, manuscript writing and revisions of the article.

The authors declare no conflicts of interest arising from the conclusions of this research. 


\section{References}

1. Zhang S, Li T-S, Soyama A, et al. (2016) Up-regulated extracellular matrix components and inflammatory chemokines may impair the regeneration of cholestatic liver. Sci Rep 6, 26540.

2. Colares JR, Schemitt EG, Hartmann RM, et al. (2016) Antioxidant and anti-inflammatory action of melatonin in an experimental model of secondary biliary cirrhosis induced by bile duct ligation. World J Gastroenterol 22, 8918.

3. Kabirifar R, Safari F, Karimollah A, et al. (2017) Quercetin protects liver injury induced by bile duct ligation via attenuation of Rac1 and NADPH oxidase 1 expression in rats. Hepatobiliary Pancreat Dis Int 16, 88-95.

4. Saxena NK, Ikeda K, Rockey DC, et al. (2002) Leptin in hepatic fibrosis: evidence for increased collagen production in stellate cells and lean littermates of ob/ob mice. Hepatology 35, 762-771.

5. García-Ruiz I, Gómez-Izquierdo E, Díaz-Sanjuán T, et al. (2012) Sp1 and Sp3 transcription factors mediate leptininduced collagen $\alpha 1$ (I) gene expression in primary culture of male rat hepatic stellate cells. Endocrinology 153, 5845-5856.

6. Kabirifar R, Safari F, Karimollah A, et al. (2017) Hepatoprotective effects of curcumin in rats after bile duct ligation via downregulation of Rac1 and NOX1. Nutrition 36, 72-78.

7. Brandes RP, Weissmann N \& Schröder K (2014) Nox family NADPH oxidases: molecular mechanisms of activation. Free Radic Biol Med 76, 208-226.

8. Zhao Y, Ma X, Wang J, et al. (2014) Curcumin protects against CCl4-induced liver fibrosis in rats by inhibiting HIF-1 $\alpha$ through an ERK-dependent pathway. Molecules 19, 18767-18780.

9. Toriumi K, Horikoshi Y, Osamura RY, et al. (2013) Carbon tetrachloride-induced hepatic injury through formation of oxidized diacylglycerol and activation of the PKC/NF-[kappa] B pathway. Lab Invest 93, 218.

10. Meshkani R \& Adeli K (2009) Hepatic insulin resistance, metabolic syndrome and cardiovascular disease. Clin Biochem 42, 1331-1346.

11. O'Shea JJ, Schwartz DM, Villarino AV, et al. (2015) The JAK-STAT pathway: impact on human disease and therapeutic intervention. Annu Rev Med 66, 311-328.

12. Elliott J \& Johnston JA (2004) SOCS: role in inflammation, allergy and homeostasis. Trends Immunol 25, 434-440.

13. Croker BA, Kiu H \& Nicholson SE (2008) SOCS regulation of the JAK/STAT signalling pathway. Semin Cell Dev Biol 19, 414-422.

14. Taniguchi CM, Ueki K \& Kahn CR (2005) Complementary roles of IRS-1 and IRS-2 in the hepatic regulation of metabolism. J Clin Invest 115, 718.

15. Rui L, Yuan M, Frantz D, et al. (2002) SOCS-1 and SOCS-3 block insulin signaling by ubiquitin-mediated degradation of IRS1 and IRS2. J Biol Chem 277, 42394-42398.

16. Tilg H \& Moschen AR (2008) Insulin resistance, inflammation, and non-alcoholic fatty liver disease. Trends Endocrinol Metab 19, 371-379.

17. Kawaguchi T, Taniguchi $\mathrm{E}$, Itou $\mathrm{M}$, et al. (2011) Insulin resistance and chronic liver disease. World J Hepatol 3, 99.

18. Leclercq IA, Morais ADS, Schroyen B, et al. (2007) Insulin resistance in hepatocytes and sinusoidal liver cells: mechanisms and consequences. J Hepatol 47, 142-156.

19. Gual P, Le Marchand-Brustel Y \& Tanti J-F (2005) Positive and negative regulation of insulin signaling through IRS-1 phosphorylation. Biochimie 87, 99-109.

20. El Swefy S, Hasan RA, Ibrahim A, et al. (2016) Curcumin and hemopressin treatment attenuates cholestasis-induced liver fibrosis in rats: role of CB1 receptors. Naunyn Schmiedebergs Arch Pharmacol 389, 103-116.
21. Wang M-E, Chen Y-C, Chen I-S, et al. (2012) Curcumin protects against thioacetamide-induced hepatic fibrosis by attenuating the inflammatory response and inducing apoptosis of damaged hepatocytes. J Nutr Biochem 23, 1352-1366.

22. Park EJ, Jeon CH, Ko G, et al. (2000) Protective effect of curcumin in rat liver injury induced by carbon tetrachloride. J Pharm Pharmacol 52, 437-440.

23. Reyes-Gordillo K, Segovia J, Shibayama M, et al. (2008) Curcumin prevents and reverses cirrhosis induced by bile duct obstruction or CCl4 in rats: role of TGF-beta modulation and oxidative stress. Fundam Clin Pharmacol 22, 417-427.

24. Afrin MR, Arumugam S, Rahman MA, et al. (2017) Curcumin reduces the risk of chronic kidney damage in mice with nonalcoholic steatohepatitis by modulating endoplasmic reticulum stress and MAPK signaling. Int Immunopharmacol 49, 161-167.

25. Gupta A, Singh VK, Kumar D, et al. (2017) Curcumin-3,4Dichloro Phenyl Pyrazole (CDPP) overcomes curcumin's low bioavailability, inhibits adipogenesis and ameliorates dyslipidemia by activating reverse cholesterol transport. Metabolism 73, 109-124.

26. Jaiswal SK, Sharma A, Gupta VK, et al. (2016) Curcumin mediated attenuation of carbofuran induced oxidative stress in rat brain. Biochem Res Int 2016, 7637931.

27. Haddadian Z, Eftekhari G, Mazloom R, et al. (2013) Effect of endotoxin on heart rate dynamics in rats with cirrhosis. Auton Neurosci 177, 104-113.

28. Ma Z, Zhang Y, Huet P-M, et al. (1999) Differential effects of jaundice and cirrhosis on $\beta$-adrenoceptor signaling in three rat models of cirrhotic cardiomyopathy. J Hepatol 30, 485-491.

29. Yagi K (1984) [39] Assay for blood plasma or serum. Methods Enzymol 105, 328-331.

30. Gülçin İ (2006) Antioxidant activity of caffeic acid (3, 4-dihydroxycinnamic acid). Toxicology 217, 213-220.

31. Tahir I, Khan MR, Shah NA, et al. (2016) Evaluation of phytochemicals, antioxidant activity and amelioration of pulmonary fibrosis with Phyllanthus emblica leaves. BMC Complement Altern Med 16, 406.

32. Matthews D, Hosker J, Rudenski A, et al. (1985) Homeostasis model assessment: insulin resistance and $\beta$-cell function from fasting plasma glucose and insulin concentrations in man. Diabetologia 28, 412-419.

33. Vivoli E, Maira G \& Marra F (2016) Liver fibrosis and leptin. Curr Pathobiol Rep 4, 69-76.

34. García-Compeán D, González-González JA, Lavalle-González FJ, et al. (2016) Hepatogenous diabetes: Is it a neglected condition in chronic liver disease? World J Gastroenterol 22, 2869.

35. Karadeniz G, Acikgoz S, Tekin IO, et al. (2008) Oxidized lowdensity-lipoprotein accumulation is associated with liver fibrosis in experimental cholestasis. Clinics 63, 531-540.

36. Tahan G, Akin H, Aydogan F, et al. (2010) Melatonin ameliorates liver fibrosis induced by bile-duct ligation in rats. Can J Surg 53, 313-318.

37. Svegliati Baroni G, D'Ambrosio L, Ferretti G, et al. (1998) Fibrogenic effect of oxidative stress on rat hepatic stellate cells. Hepatology 27, 720-726.

38. Qiao Q, Le Manach S, Sotton B, et al. (2016) Deep sexual dimorphism in adult medaka fish liver highlighted by multiomic approach. Sci Rep 6, 32459.

39. George J (2003) Ascorbic acid concentrations in dimethylnitrosamine-induced hepatic fibrosis in rats. Clin Chim Acta 335, 39-47.

40. Trebicka J, Hennenberg M, Odenthal M, et al. (2010) Atorvastatin attenuates hepatic fibrosis in rats after bile duct ligation via decreased turnover of hepatic stellate cells. J Hepatol 53, 702-712.

41. Reyes-Gordillo K, Segovia J, Shibayama M, et al. (2007) Curcumin protects against acute liver damage in the rat by 
inhibiting NF- $\kappa \mathrm{B}$, proinflammatory cytokines production and oxidative stress. Biochim Biophys Acta 1770, 989-996.

42. Bahçecioğlu İH, Yalniz M, Ataseven H, et al. (2004) TNF- $\alpha$ and leptin in experimental liver fibrosis models induced by carbon tetrachloride and by common bile duct ligation. Cell Biochem Funct 22, 359-363.

43. Choi SS, Sicklick JK, Ma Q, et al. (2006) Sustained activation of Rac1 in hepatic stellate cells promotes liver injury and fibrosis in mice. Hepatology 44, 1267-1277.

44. Li J, Zhu H, Shen E, et al. (2010) Deficiency of rac1 blocks $\mathrm{NADPH}$ oxidase activation, inhibits endoplasmic reticulum stress, and reduces myocardial remodeling in a mouse model of type 1 diabetes. Diabetes 59, 2033-2042.
45. Hafez MM, Al-Harbi NO, Al-Hoshani AR, et al. (2015) Hepatoprotective effect of rutin via IL-6/STAT3 pathway in CCl 4-induced hepatotoxicity in rats. Biol Res 48, 30.

46. Kim Y, Kim Y, Cho Y, et al. (2012) Metformin ameliorates IL-6-induced hepatic insulin resistance via induction of orphan nuclear receptor small heterodimer partner (SHP) in mouse models. Diabetologia 55, 1482-1494.

47. El-Zayadi A-R (2010) Insulin resistance. Arab J Gastroenterol 11, 66-69.

48. Sadek K, Beltagy D, Saleh E, et al. (2016) Camel milk and bee honey regulate profibrotic cytokine gene transcripts in liver cirrhosis induced by carbon tetrachloride. Can J Physiol Pharmacol 94, 1141-1150. 